\title{
Super-adherencia del maratoniano: variables predictoras y diferencias de género
}

\author{
Super-Adherence of Marathoners: Predictor \\ Variables and Gender Differences
}

Recibido: febrero 15 de 2010 | Revisado: julio 31 de 2010 | Aceptado: noviembre 31 de 2010

\author{
ANTONIO ZARAUZ SANCHO* \\ I.E.S. Azcona de Almería, España \\ FRANCISCO RUIZ-JUAN ** \\ Universidad de Murcia, España
}

\section{RESUMEN}

La vertiente lúdica del creciente tiempo de ocio en España cada vez más se está rellenando con el postmodernista fenómeno de la popularización de la carrera continua. El presente estudio investiga en su mayor practicante: el maratoniano qué variables motivacionales y de entrenamiento predicen su elevado compromiso y adicción negativa con su actividad deportiva, con el objetivo de tratar de obtener unas conclusiones útiles para aumentar la adherencia a programas de actividad físico-deportiva en la población general. Para esto, se parte de la hipótesis de que los maratonianos de nuestra población tienen unos niveles muy altos de compromiso y adicción negativa a correr, que ambos constructos tienen una elevada correlación entre sí y se pueden predecir tanto por sus altos y diferentes tipos de motivación, como por cuatro variables de entrenamiento. Los resultados confirman las hipótesis, obteniéndose valiosos datos descriptivos y unos sólidos modelos predictivos en ambos constructos evaluados por sexo. Sin embargo, lo que fue difícil de explicar fue que las mujeres obtuvieron grados más altos que los hombres en compromiso, adicción negativa a correr y en todas las motivaciones, muchas veces además de forma significativa.

Palabras clave autores:

Compromiso, adicción, motivación, maratón.

Palabras clave descriptores:

Adherencia, actividad física, psicología del deporte.

\section{A B S T R A C T}

The playful side of the growing leisure time in Spain is increasingly being filled more with the postmodern phenomenon of popularization of continuous running. This study investigates in the most practitioner: the marathoner, what training and motivational variables predict their high commitment and negative addiction with their sport, with the aim of trying to get some conclusions useful for increasing adherence to physical activity programs sport in the general population. To do we start from the assumption that the marathoners of our population have very high levels of commitment and negative addiction to running, the two constructs are highly correlated with each other and can be predicted both by high and different types of motivation, as by four training variables. The results obtained confirm the hypothesis, valuable descriptive data and solid in both constructs predictive models assessed by sex. However, what we failed to explain was that women got higher grades than men in commitment, negative addiction to running and all motivations, often significantly further. Key words authors:

Commitment, addiction, motivation, marathon.

Key words plus:

Adherence, physical activity, Sport Psychology. 


\section{Introducción}

En los últimos años, la falta de tiempo ha sido el motivo más aludido para la inactividad física (Valero, Gómez, Gavala, Ruiz-Juan \& García, 2007), aunque cada vez va cobrando menos fuerza en nuestra actual sociedad postmodernista, pues el tiempo de ocio es cada vez mayor y una de las vertientes en la que se está empleando más es en la lúdica, con la práctica de deportes modernos y tradicionales, hobbies, recreación en el hogar, juegos, etc. Ejemplo de estas prácticas deportivas es el jogging o carrera continua, que empezó a popularizarse de manera generalizada en la década de los 70 en EE. UU. (Glasser, 1976; Kostrubala, 1977), así como la figura de su practicante por excelencia: el maratoniano. Este fenómeno de la popularización masiva de la carrera continua llegó a España durante la pasada década de los 90 (García, 2005), probablemente por la influencia del postmodernismo en la sociedad y la concesión de los Juegos Olímpicos en 1992 a Barcelona.

Si se mira el calendario de competiciones de los últimos años en España, se corrobora que cada vez hay más ayuntamientos que organizan una maratón, y en las propias web de cada carrera también se observa que no cesa de aumentar rápidamente el número de inscritos a esta modalidad deportiva. En la edición de 2009 de la maratón de Barcelona, por ejemplo, cerca de 10.000 personas cruzaron la meta, a pesar de que tan solo para acabar una maratón se requiere una capacidad cardiorespiratoria y un nivel de resistencia muscular y psicológica tan alto que solo puede adquirirse tras muchos meses o años de constante entrenamiento.

Por esto, los maratonianos son probablemente los deportistas con un mayor grado de compromiso con su actividad deportiva (Carmack \& Martens, 1979), dado que no solo entrenan casi todos los días de la semana, sino que se mantienen en su práctica durante muchos años. Este es el primero de los constructos teóricos que se va a investigar en este estudio: el compromiso a correr (CC). Etimológicamente, compromiso es sencillamente una 'obligación contraída' (Real Academia Española, 2004), y las dos variables que definen con claridad un alto CC en la población de maratonianos son la mayor cantidad de días de entrenamiento y los kilómetros entrenados semanalmente (Thornton $\&$ Scott, 1995).

Sin embargo, este compromiso de los maratonianos puede ser llevado a límites que van en detrimento de su calidad de vida (Ardila, 2003), pues llega a deteriorarse la vida social del corredor, su trabajo o incluso su salud; a nivel físico: seguir corriendo lesionado en contra del consejo del médico, fatiga, disminución del rendimiento (Meeusen et al., 2006), o a nivel mental: síndrome de abstinencia, ansiedad e irritabilidad cuando se pierde un entrenamiento (Pargman, 1980). Es entonces cuando aquel compromiso pasa a convertirse en el segundo constructo teórico objeto de nuestro estudio: la adicción negativa a correr (ANC).

Glasser (1976) también definió la adicción positiva a correr (APC), entendiéndola como una actividad que incrementa la fuerza mental y cuando se pierde produce algún tipo de sufrimiento o malestar (físico o psicológico). Para diferenciar ambas adicciones, nos explicaba que la APC es agradable, pero no domina la vida de una persona, como ocurre en el caso de la ANC, por lo que esta última podría ser comparable con la adicción a las compras de manera compulsiva e irreflexiva que explica I. García (2007).

La APC se equipara al concepto de CC, señalan Carmack y Martens (1979) en un estudio donde, además de desarrollar y validar un instrumento para medir el mencionado CC (la Commitment to Running Scale [CR]), añaden que existe una sensación placentera de euforia asociada a la APC que se consigue al correr desconectando. En español, existe una versión de la CR con adecuadas propiedades psicométricas, la CR-11 de Ruiz-Juan y Zarauz (2011a).

Para la medida de la ANC, Chapman y De Castro (1990) desarrollaron y validaron la Running Addiction Scale (RAS), instrumento específico de referencia hasta la fecha. En su estudio, además, concluyeron que ambos sexos podían tener mucho $\mathrm{CC}$, pero no por esto las mujeres daban valores altos de ANC como hacían los hombres. También existe una versión en español de la RAS con adecuadas 
propiedades psicométricas, la RAS-8 de Zarauz y Ruiz-Juan (2011).

Por el contrario, investigaciones más recientes (Dawson \& Peco, 2004; Thornton \& Scott, 1995) encontraron que sí existía una alta correlación entre el CC y ANC en ambos sexos, concluyendo que la segunda suele ser como consecuencia del primero. Por esto, Thornton y Scott (1995) advirtieron que las consecuencias de la ANC no debían tomarse a la ligera, pues los corredores con propensión al estrés que se iniciaban a la carrera continua como método de mejorar su salud, podrían desarrollar un comportamiento patológico obsesivo con su $\mathrm{CC}$, aspecto este corroborado posteriormente por Leedy (2000).

A esta particular manera de los maratonianos de tomarse su actividad deportiva, suma de sus elevados grados de CC y ANC, Masters, Ogles y Jolton (1993) la denominaron "súper-adherencia", pues la mayoría de estos corredores entrenan mucho más de lo necesario para mantener la forma (que es lo que hacen la mayoría de los deportistas) y continúan a ese ritmo de trabajo durante muchos años.

Por tanto, cabe preguntarse iqué es lo que mueve a este creciente número de personas a tener esta superadherencia que asumen para preparar un maratón: el reto que supone hacerlo por primera vez, mejorar su tiempo previo si ya se ha terminado alguno, la salud, la amistad con el grupo de amigos con los que entrena y compite, adelgazar, estar en forma, evadirse de las preocupaciones diarias mientras corre...? Estamos ante un tercer constructo teórico, las motivaciones, que se va a investigar en este estudio por su influencia decisiva en los dos primeros (CC y ANC).

Para medir la motivación específica para correr de los maratonianos, Masters et al. (1993) desarrollaron y validaron un instrumento referente hasta la fecha: las Motivations of Marathoners Scales (MOMS), que numerosos investigadores han utilizado o se han basado en él para crear los suyos propios. Contiene 56 motivos para correr, que a su vez se agrupan en cuatro categorías generales de motivos (Motivos Psicológicos, de Logro, Sociales y de Salud Física) y estas a su vez se pueden agrupar en nueve categorías o escalas específicas de mo- tivaciones (Salud general, Concerniente al peso, Afiliación, Reconocimiento, Competición, Logro de meta personal, Metas psicológicas, Autoestima y Sentido de la vida). Recientemente, Ruiz-Juan y Zarauz (2011b), al realizar una adaptación al español de las MOMS, obtuvieron una escala con adecuadas propiedades psicométricas, la MOMS-34, donde los 56 motivos de la escala original se reducían a 34 en la población española de maratonianos, y las nueve escalas de motivaciones a siete.

Estos tres constructos teóricos definidos hasta ahora (CC, ANC y motivación) están influenciados y se pueden predecir por una serie de variables, como las que consideran Masters et al. (1993) en su Demographic and Training Questionnaire, donde enumeran un buen número de variables sociodemográficas, de entrenamiento y de rendimiento.

Así, tenemos que los corredores con un mayor grado de CC (= APC) son varones, con más de un año de dedicación a la carrera continua en un mínimo de tres sesiones semanales de al menos 40 minutos (Carmack \& Martens, 1979), promediando hombres y mujeres respectivamente 43.8 y 31.4 minutos por entrenamiento, 5.6 y 5 días de entrenamiento a la semana y 6.3 y 1.8 años de experiencia corriendo. Estos autores, además, compararon los motivos de inicio y continuación de los 75 maratonianos con mayor CC, y en todos los casos las motivaciones de Salud física y psicológica, seguida del Logro de objetivos, fueron las motivaciones más aludidas por su población.

Por su parte, Thornton y Scott (1995) obtuvieron que el 77 \% de los maratonianos de su población tenía unos niveles tan altos de CC que les hacía tener ANC, siendo el Dominio y el Reconocimiento social las motivaciones que más preveían esa súperadherencia.

En resumen, hasta ahora se han presentado una serie de complejas influencias y relaciones entre tres constructos teóricos entre sí (CC, ANC y motivación) y con un gran número de variables sociodemográficas, de entrenamiento y rendimiento (Figura 1).

Sin embargo, aunque estas relaciones han servido de inspiración y base para la presente investigación, en el estudio solo se consideraron 
las relaciones entre estos tres constructos teóricos entre sí y con las variables más repetidas en la bibliografía, tanto a nivel de entrenamiento (promedios de kilómetros entrenados por semana, horas por entrenamiento, días de entrenamiento por semana y años corriendo), como sociodemográfico (sexo).

Como objetivo de nuestra investigación se planteó analizar estas relaciones en su conjunto para tratar de comprender el porqué de esta superadherencia de los maratonianos (Carmack \& Martens, 1979; Dawson \& Peco, 2004; Masters et al., 1993; Thornton \& Scott, 1995). Al hacerlo, se extraerían unas conclusiones que quizá puedan servir de punto de partida a los profesionales de la actividad física a la hora de diseñar programas de trabajo lo suficientemente motivantes en otras poblaciones, para que disminuya la alta inactividad física de la población general (Gómez, RuizJuan, García, Flores \& Barbero, 2008) y su alta tasa de abandono deportivo en los primeros meses (Moreno, Martínez \& Alonso, 2006), alargando su ciclo de vida activa y luchando contra el abandono deportivo, aspecto muy relacionado con el sedentarismo y el riesgo de padecer enfermedades crónicas y degenerativas (Fohelholm, Stallknecht \& Van Baak, 2006; Vuori, 2004). Esto último, unido a que todavía no se han estudiado las relaciones entre CC, ANC y motivaciones en la población de maratonianos españoles, serviría para completar la justificación del presente estudio.

Se partió de la hipótesis de que los maratonianos tienen unos niveles muy altos de CCy ANC, además de que estos dos constructos tienen una elevada correlación entre sí y se pueden predecir por variables diferentes en hombres y mujeres (por sus altos y diferentes tipos de motivación y por los promedios de kilómetros entrenados por semana, horas por entrenamiento, días de entrenamiento por semana y años corriendo). También se presumió que, aunque los promedios que

\section{Variables de Entrenamiento y Rendimiento:}

Promedios de kilómetros entrenados a la semana, de tiempo por entrenamiento, de días entrenados a la semana y de compañeros de entrenamiento, porcentaje de tiempo que entrena solo a la semana, anos corriendo, tener entrenador, número de maratones terminados, mejor marca en maratón y promedio de marcas en los maratones terminados.

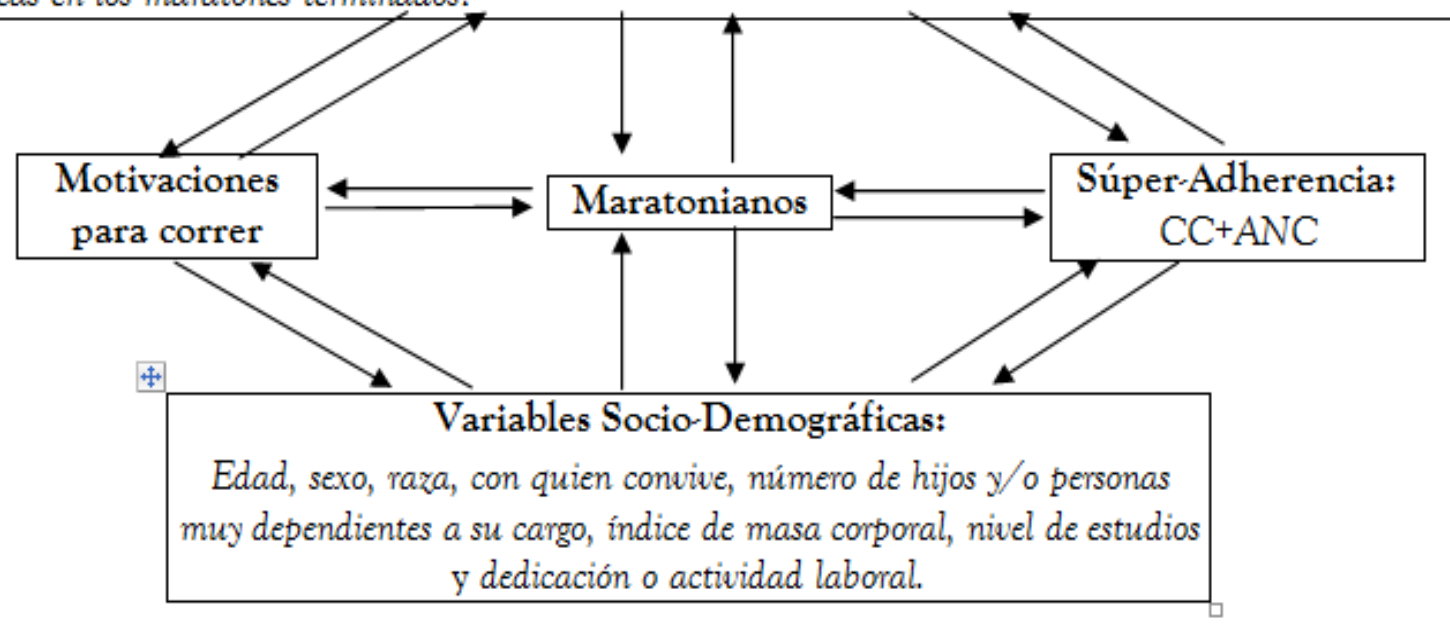

Figura 1. Relaciones entre constructos y variables en los maratonianos.

Fuente: elaboración propia. 
se obtengan en estas cuatro variables de entrenamiento van a aumentar considerablemente respecto a los que resultaron en estudios precedentes, siguen existiendo notables diferencias entre hombres y mujeres a favor de los primeros, tanto en estos promedios de las variables descriptivas de entrenamiento como en los del CC, la ANC y las motivaciones.

\section{Método}

\section{Participantes}

Se partió del total de maratonianos inscritos en las últimas ediciones de las maratones de San Sebastián, Sevilla y Barcelona. Para asegurar que la muestra fuera representativa (error $\pm 3 \%$, intervalo de confianza $95.5 \%$ ), se empleó un diseño de muestreo estratificado por afijación proporcional teniendo en cuenta el sexo (88.94\% hombres; $11.05 \%$ mujeres) y la edad.

Se administró un cuestionario a 1.226 maratonianos que corrieron las maratones de San Sebastián (30-11-08), Sevilla (22-02-09) y Barcelona (01-03-09). La muestra aleatoria estaba compuesta por 1.108 hombres $(90.37 \%)$ con rango de edad de 17 a 71 años $(M=39.92 ; D E=8.91)$ y $118 \mathrm{mu}$ jeres $(9.63 \%)$ con rango de edad de 21 a 57 años $(\mathrm{M}=38.10 ; \mathrm{DE}=8.02)$.

\section{Instrumentos}

CR-11 (Ruiz-Juan Ë Zarauz, 2011a).

Versión española validada (Apéndice

1) de la Commitment to Running Scale

(CR) de Carmack y Martens (1979).

Contiene 11 ítems para medir el CC. Las respuestas se recogen a través de una escala tipo Likert desde 1 (no está nada de acuerdo) hasta 5 (está totalmente de acuerdo), de manera que se estaría entre una puntuación mínima en la CR-11 de 11 (mínimo CC) y máxima de 55 (máximo CC). La consistencia interna de la escala es de $\boldsymbol{a}=0.87$. Presenta valores correctos que permiten determinar una aceptable bondad de ajuste del modelo original. Los resultados del AFC fueron: $x^{2} / g l=2.93$; GFI = 0.91; IFI
$=0.93 ; \mathrm{CFI}=0.93 ; \mathrm{TLI}=0.91 ; \mathrm{SRMR}=0.05$; RMSEA $=0.05$.

RAS-8 (Zarauz E8 Ruiz-Juan, 2011). Versión española validada (Apéndice 2) de la Running Addiction Scale (RAS) de Chapman y De Castro (1990).

Contiene ocho ítems para medir la ANC. Las respuestas de los evaluados se recogen a través de una escala tipo Likert desde 1 (no está nada de acuerdo) hasta 7 (está totalmente de acuerdo), de manera que se presenta entre una puntuación mínima en la RAS-8 de ocho (mínima ANC) y máxima de 56 (máxima ANC). La consistencia interna de la escala es de $\boldsymbol{a}=0.84$. Presenta valores correctos que permiten determinar una aceptable bondad de ajuste del modelo original. Los resultados del AFC fueron: $X^{2} / g l=4.07$ GFI $=0.88$; $\mathrm{IFI}=0.9 ; \mathrm{CFI}=0.9 ; \mathrm{TLI}=0.88 ; \mathrm{SRMR}=0.05$; RMSEA $=0.07$.

MOMS-34 (Ruiz-Juan Eु Zarauz, 2011b). Versión española validada (Apéndice 3) de la Motivations of Marathoners Scales (MOMS) de Masters et al. (1993).

Contiene siete escalas de motivaciones con 34 ítems o motivos para correr, que miden el grado de orientación motivacional para correr por una u otra escala. Las respuestas se recogen a través de una escala tipo Likert desde 1 (no es una razón para correr) hasta 7 (es una razón muy importante para correr), de manera que en cada escala se presenta entre una puntuación media mínima de 1 (mínima motivación para correr por esa escala) a 7 (máxima motivación para correr por esa escala). La consistencia interna de las escalas osciló entre un valor mínimo de $a=0.8$ (Orientación a la salud) y máximo de $a=0.9$ (Reconocimiento). $\mathrm{El}$ instrumento presenta valores correctos que permiten determinar una aceptable bondad de ajuste del modelo original. Los resultados del AFC fueron: $X^{2}{ }_{(506)}=1584.677 ; p<0.001 ; X^{2} / g l=3.13$; $\mathrm{GFI}=0.89 ; \mathrm{IFI}=0.91 ; \mathrm{CFI}=0.91 ; \mathrm{TLI}=0.9$; SRMR $=0.06 ;$ RMSEA $=0.07$. 


\section{Variables de entrenamiento}

Promedios de kilómetros entrenados a la semana, de tiempo por entrenamiento, de días entrenados a la semana y de años corriendo.

\section{Procedimiento}

Se pidió permiso a la organización de las tres carreras mediante una carta en la que se explicaban los objetivos de la investigación y cómo se iba a realizar, acompañándose un modelo del instrumento. Se concedió el permiso y se facilitó un stand propio al efecto. El cuestionario fue administrado por los investigadores durante la recogida de dorsales de los atletas participantes, durante los momentos de esparcimiento y relax de la visita a la Feria del Corredor el día previo a la carrera. Todos los sujetos fueron informados del objetivo del estudio, de la voluntariedad, absoluta confidencialidad de las respuestas y manejo de los datos, de que no había respuestas correctas o incorrectas y se les solicitó que contestaran con la máxima sinceridad y honestidad.

\section{Análisis estadísticos de los datos}

Los análisis de ítems, homogeneidad de las escalas, estructura interna, correlación entre las escalas (coeficiente de Pearson), consistencia interna (alfa de Cronbach), diferencia de medias por sexo ( $t$ de Student) y regresión lineal se realizaron con el SPSS 17.0. Para evaluar la estructura factorial se realizó un análisis factorial confirmatorio a través del software AMOS 18.0.

\section{Resultados}

\section{Descriptivos y diferencias por sexo}

La puntuación media obtenida en la CR-11 fue de 3.84 (sobre 5) con una desviación estandar de 0.71 , siendo de 3.82 en varones $(D E=0.7)$ y de 4.04 en mujeres $(D E=0.69)$. Por su parte, en la RAS-8 la puntuación media obtenida fue de 4.95 (sobre 7) $(\mathrm{DE}=0.92)$ para el total de la muestra y de $4.94(D E=0.92)$ y $5.06(D E=0.95)$, respec- tivamente para varones y mujeres. Los resultados de la prueba $t$ (Tabla 1) indican la inexistencia de diferencias significativas en ANC (RAS-8) entre hombres y mujeres, pero si en CC (CR-11) a favor de las mujeres $(F=0.026, p<0.01)$. Además, la correlación entre ambas escalas, CR-11 y RAS-8, es alta, positiva y significativa $(r=0.59)$ y sin diferencias entre géneros.

Las puntuaciones medias de las siete escalas de motivaciones de las MOMS-34, ordenadas de mayor a menor son las siguientes. Significado de la vidaAutoestima: $M=4.73(D E=1.29)$; Orientación a la salud: $M=4.72(D E=1.38)$; Superación de metas personales-Competición: $M=4.39(\mathrm{DE}=1.34)$; Meta psicológica: $M=4.34(D E=1.58)$; Afiliación: $M=3.82(D E=1.54) ;$ Peso: $M=3.78(D E=1.63) y$ Reconocimiento: $\mathrm{M}=2.80(\mathrm{DE}=1.44)$. Al realizar la prueba $t$ de Student para ver las diferencias de sexo (Tabla 2), se aprecian diferencias significativas en las escalas de Significado de la vida-Autoestima, Metas psicológicas, Afiliación y Peso, siempre a favor de las mujeres. En el resto de escalas (Orientación a la Salud, Metas Personales-Competición y Reconocimiento) las diferencias de puntuaciones medias entre hombres y mujeres no son significativas.

En las variables independientes continuas que se manejaron como hipotéticamente predictoras del CC y/o ANC, es decir, sus hábitos de entrenamiento, no se encontraron diferencias significativas según el sexo, con la excepción de los kilómetros entrenados a la semana, siendo los valores significativamente mayores en los hombres. De esta manera, los maratonianos de la muestra promedian 54.71 kilómetros de entrenamiento a la semana frente a los 45.78 de las maratonianas. Igualmente, ambos sexos sobrepasan la hora y diez minutos por entrenamiento, con una media de más de cuatro días de entrenamiento semanales y llevan entre nueve y diez años corriendo (Tabla 1).

\section{Análisis regresivo}

A continuación, se realizó un análisis de regresión lineal multivariado tomando como variables dependientes las puntuaciones medias de la CR-11 o RAS- 8 y como variables predictoras las puntua- 
TABLA 1

Media, desviación típica, F y significatividad del CC, ANC y de las variables de entrenamiento

\begin{tabular}{|c|c|c|c|c|}
\hline Variables & Sexo & $M$ & $D E$ & $\mathrm{~F}+$ significatividad \\
\hline \multirow{2}{*}{ CC } & Hombres & 3.82 & 0.7 & \multirow{2}{*}{$0.026^{* *}$} \\
\hline & Mujeres & 4.04 & 0.69 & \\
\hline \multirow{2}{*}{ ANC } & Hombres & 4.94 & 0.92 & \multirow{2}{*}{0.089} \\
\hline & Mujeres & 5.06 & 0.95 & \\
\hline \multirow{2}{*}{$\mathrm{Km} / \mathrm{semana}$} & Hombres & 54.71 & 23.86 & \multirow{2}{*}{$4 * * *$} \\
\hline & Mujeres & 45.78 & 18.7 & \\
\hline \multirow{2}{*}{ Horas entrenamiento/sesión } & Hombres & 1.18 & 0.5 & \multirow{2}{*}{3} \\
\hline & Mujeres & 1.11 & 0.38 & \\
\hline \multirow{2}{*}{ Días entrenamiento/semana } & Hombres & 4.31 & 1.14 & \multirow{2}{*}{0.05} \\
\hline & Mujeres & 4.24 & 1.13 & \\
\hline \multirow{2}{*}{ Años corriendo } & Hombres & 9.9 & 8.09 & \multirow{2}{*}{4.75} \\
\hline & Mujeres & 8.92 & 6.83 & \\
\hline
\end{tabular}

$* * p<0.01 ; * * p<0.001$. Hombres $(N=1.108)$; Mujeres $(N=118)$

Fuente: elaboración propia.

TABLA 2

Media, desviación típica, F y significación de las 7 escalas de las MOMS-34

\begin{tabular}{lcccc}
\hline \multicolumn{1}{c}{ Escalas de Motivaciones } & Sexo & $M$ & $D E$ & F+significatividad \\
\hline \multirow{2}{*}{ Significado de la vida-Autoestima } & Hombres & 4.7 & 1.29 & \multirow{2}{*}{$0.23^{* * *}$} \\
\hline \multirow{2}{*}{ Orientación a la salud } & Mujeres & 5.05 & 1.23 & \\
\hline \multirow{2}{*}{ Superación de metas personales-Competición } & Hombres & 4.72 & 1.38 & \multirow{2}{*}{0.02} \\
& Mujeres & 4.78 & 1.38 & \\
\hline \multirow{2}{*}{ Metas psicológicas } & Hombres & 4.39 & 1.34 & \multirow{2}{*}{0.12} \\
\hline \multirow{2}{*}{ Afiliación } & Mujeres & 4.41 & 1.27 & \multirow{2}{*}{$3.82^{* * *}$} \\
\hline \multirow{2}{*}{ Peso } & Hombres & 4.28 & 1.56 & \multirow{2}{*}{$0.34^{*}$} \\
\hline \multirow{2}{*}{ Reconocimiento } & Mujeres & 4.85 & 1.71 & \multirow{2}{*}{$0.08^{*}$} \\
\hline
\end{tabular}

$* p<0.05 ; * * p<0.01 ; * * * p<0.001$. Hombres $(N=1108)$ y mujeres $(N=118)$

Fuente: elaboración propia.

ciones medias en las motivaciones y en las variables de entrenamiento. Por último, como variable de selección se consideró el ser hombre o mujer.

Como resultado de este análisis se obtuvieron unos sólidos modelos que explicaban una gran parte de la varianza en hombres y en mujeres, llegando en algún caso a sobrepasar el $40 \%$. De este análisis, se extrajo el valor de $R^{2}$ para explicar la varianza, el de Beta para explicar la predicción entre variables, el de $F$ para ver si existe relación entre las variables seleccionadas y su nivel de significancia (Tabla 3).

Los modelos muestran que el CC en hombres, se puede predecir significativamente por su mayor número de años corriendo y de kilómetros 
TABLA 3

Análisis regresivo lineal multivariado. Modelos que predicen significativamente el CC y ANC por género en función de las motivaciones y cuatro variables de entrenamiento

\begin{tabular}{|c|c|c|c|c|c|c|c|c|}
\hline & \multicolumn{4}{|c|}{$\mathrm{CC}$} & \multicolumn{4}{|c|}{ ANC } \\
\hline & \multicolumn{2}{|c|}{ Hombres } & \multicolumn{2}{|c|}{ Mujeres } & \multicolumn{2}{|c|}{ Hombres } & \multicolumn{2}{|c|}{ Mujeres } \\
\hline & Beta & Sign. & Beta & Sign. & Beta & Sign. & Beta & Sign. \\
\hline $\mathrm{Km} . / \mathrm{semana}$ & 0.164 & $* * *$ & 0.207 & & 0.122 & $* *$ & 0.028 & \\
\hline Horas/entrenamiento & 0 & & -0.021 & & -0.027 & & 0.077 & \\
\hline Días entr./semana & 0.038 & & 0.046 & & 0.171 & $* * *$ & 0.19 & \\
\hline Años corriendo & 0.083 & $* *$ & 0.036 & & 0.011 & & -0.152 & \\
\hline Salud & 0.155 & $* * *$ & 0.12 & & 0.15 & $* * *$ & 0.172 & \\
\hline Peso & -0.001 & & -0.053 & & 0.006 & & 0.104 & \\
\hline Sup. Metas-Comp. & 0.061 & & -0.026 & & 0.133 & $* * *$ & 0.029 & \\
\hline Reconocimiento & -0.143 & $* * *$ & -0.161 & & -0.179 & $* * *$ & -0.353 & $* *$ \\
\hline Afiliación & 0.001 & & -0.007 & & -0.002 & & -0.094 & \\
\hline Metas psicológicas & 0.128 & $* * *$ & 0.275 & $* *$ & 0.05 & & 0.186 & \\
\hline \multirow[t]{2}{*}{ Sign. Vida-Autoestima } & 0.325 & $* * *$ & 0.348 & $* *$ & 0.158 & $* * *$ & 0.255 & * \\
\hline & \multicolumn{2}{|c|}{$\begin{array}{l}R^{2}=0.317 \\
F=46.233\end{array}$} & \multicolumn{2}{|c|}{$\begin{aligned} R^{2} & =0.405 \\
F & =6.553\end{aligned}$} & \multicolumn{2}{|c|}{$\begin{array}{l}R^{2}=0.198 \\
F=24.663\end{array}$} & \multicolumn{2}{|c|}{$\begin{aligned} R^{2} & =0.346 \\
F & =5.091\end{aligned}$} \\
\hline
\end{tabular}

$* p<0.05 ; * * p 0.01 ; * * p<0.001$.

Fuente: elaboración propia.

entrenados a la semana, así como por puntuar alto en las motivaciones de Significado de la vida-Autoestima, Metas Psicológicas y Salud, y bajo en las motivaciones de Reconocimiento (explicando este primer modelo el $31.7 \%$ de la varianza). Sin embargo, la predicción en mujeres sería únicamente por puntuar alto en las motivaciones de Metas psicológicas y Significado de la vida-Autoestima (explicando este modelo el $40.5 \%$ de la varianza).

Por otra parte, la ANC en hombres se puede predecir significativamente por su mayor cantidad de días y kilómetros de entrenamiento a la semana, así como por puntuar alto en las motivaciones de Salud, Superación de Metas Personales-Competición y Significado de la Vida-Autoestima y bajo en la de Reconocimiento (explicando este modelo el 19.8\% de la varianza). Sin embargo, la predicción en mujeres sería únicamente por puntuar alto en la motivación de Significado de la Vida-Autoestima y bajo en la de Reconocimiento (explicando este modelo el $34.6 \%$ de la varianza).

\section{Discusión y conclusiones}

En este estudio se partió de una muestra representativa de la población de los maratonianos españoles para investigar las relaciones entre sus constructos teóricos psicológicos propios (CC y ANC) entre sí y con una serie de variables predictoras, tanto de tipo motivacional como de hábitos de entrenamiento.

La muestra, como se pensó desde el principio, tiene unos altos grados de CC, como sucedió con la de Carmack y Martens (1979), Thornton y Scott (1995) y Dawson y Peco (2004), y también de ANC, como pasó con la de Chapman y De Castro (1990), Thornton y Scott (1995) y Dawson y Peco (2004). Sin embargo, lo que no se pudo predecir fue que las mujeres alcanzaran niveles más altos que los hombres en los mencionados constructos, sobre todo y de manera significativa en CC, pues en ninguno de los estudios citados anteriormente se produjo.

De la misma manera, tal como se hipotetizó, los maratonianos tienen unos altos niveles motivacionales con su actividad deportiva, similares a los de Masters et al. (1993), aunque lo que tampoco fue 
posible prevenir fue que las mujeres, en todos los casos, tuviesen una puntuación promedio mayor en cada una de las motivaciones, e incluso, en la mayoría de ellas, de manera significativa, pues no existen referencias de ello hasta la fecha.

En cuanto a los hábitos de entrenamiento, como se predijo, los promedios de horas por sesión de entrenamiento, de años corriendo y de kilómetros entrenados a la semana, han aumentado considerablemente respecto a los estudios precedentes (Carmack \& Martens, 1979; Chapman \& De Castro, 1990; Dawson \& Peco, 2004; Masters et al., 1993; Thornton \& Scott, 1995). Sin embargo, resultó inesperado que las mujeres tuviesen unos valores promedio similares o solo ligeramente inferiores a los varones, lo cual indica un claro cambio de tendencia para equilibrar las tradicionales diferencias significativas a favor de los varones en estas variables, como las de los pioneros en estudiar a esta población (Carmack \& Martens, 1979).

Todos estos cambios de tendencia mencionados en los hábitos de entrenamiento, por un lado, o las diferencias a favor de las mujeres respecto al grado de los tres constructos evaluados por otro, podrían estar debidos a la más rápida evolución del rol de mujer en nuestra actual sociedad postmodernista española del siglo XXI (Águila, 2005).

Como les sucedió a Thornton y Scott (1995) y Dawson y Peco (2004), la correlación entre CC y ANC en nuestros corredores es muy alta, tanto en hombres como en mujeres, aunque con Carmack y Martens (1979) y Chapman y De Castro (1990) no fue así. Esto puede deberse a la evolución de la sociedad mencionada anteriormente, puesto que nuestros resultados coinciden con los de los estudios más recientes en el tiempo. Por esto, se puede afirmar que, en la actualidad, por lo general un aumento CC conlleva un aumento en la ANC, además de que ambos conceptos son predecibles por diferentes variables en uno y otro sexo, como se pensó.

Con base en el análisis regresivo, se puede afirmar que casi todos los tipos de motivación fueron predictores tanto del CC como de la ANC en hombres, lo cual indica que las motivaciones para correr en ellos no son únicas ni excluyentes de otras, sino que normalmente son varias y altas, como se había previsto y confirma la literatura consultada (Carmack \& Martens, 1979; Dawson \& Peco, 2004; Leedy, 2000; Masters et al., 1993; Thornton \& Scott, 1995). Al igual que Carmack y Martens (1979), Leedy (2000) y Thornton y Scott (1995), las motivaciones de Peso y Afiliación no resultaron predictoras de manera significativa en ninguno de los sexos (por ser de las menos valoradas por nuestra población). Sin embargo, no fue posible prevenir que en las mujeres solo dos tipos de motivación fuesen predictoras de su CC o ANC.

No se acertó del todo en la hipótesis en cuanto a dos aspectos relacionados con los hábitos de entrenamiento. Por un lado, las horas por sesión de entrenamiento no fueron una variable predictora del CC o la ANC en hombres. Por otro lado, en el caso de las mujeres, ninguna de las variables de entrenamiento manejadas como predictoras finalmente resultaron serlo. Todo esto contrasta con todas las referencias previas (Carmack \& Martens, 1979; Chapman \& De Castro, 1990; Dawson \& Peco, 2004), haciéndose patente una vez más las evidentes diferencias entre sexos que sí se predijeron.

Con respecto a las motivaciones, tanto en los hombres como en las mujeres de la muestra, las de tipo Significado de la vida-Autoestima son las que alcanzan puntuaciones medias más altas por encima de las de Superación de Metas PersonalesCompetición, al igual que Ogles y Masters (2003), lo cual evidencia una inclinación general en esta población hacia las motivaciones intrínsecas o las relacionadas con la tarea por encima de las motivaciones extrínsecas o las relacionadas con el ego. Esta tendencia motivacional parece repetirse en deportes de características similares, como el triatlón (Ruiz Tendero, 2006), pero no en deportes individuales diferentes como por ejemplo el tenis (Villamarín, Mauri \& Sanz, 1998), donde priman más las motivaciones relacionadas con el ego y la competición.

Por el contrario, las motivaciones de Reconocimiento fueron las menos valoradas, al igual que Thornton y Scott (1995), tanto por hombres como por mujeres, pues en ambos casos no se acercaron a la puntuación media, lo cual vuelve a evidenciar 
que las motivaciones extrínsecas o las relacionadas con el ego tienen poca importancia en la mayoría de los maratonianos.

Por todo esto, como uno de los objetivos del presente estudio era tratar de sentar unas bases de las que puedan partir los profesionales de la actividad física y el deporte para aumentar el compromiso de sus alumnos, se podría concluir sugiriendo que sus programas deberían centrarse sobre todo en aumentar las motivaciones de Significado de la Vida-Autoestima y Metas Psicológicas, tanto en hombres como en mujeres, por ser las motivaciones que en ambos casos predicen un mayor CC, estando además entre las más valoradas por nuestra población. Además, también habría que reforzar la motivación de Salud, pues junto con las dos anteriores, predice un alto CC en los hombres y es la segunda más valorada por las mujeres de nuestra población.

Por contra, no interesaría reforzar las motivaciones de Superación de metas personales-Competición, pues aunque sea la tercera más valorada por nuestra población, predice en hombres la parte de la "super-adherencia" que no nos interesa adquirir: la ANC. Tampoco parece que ninguna de las variables de entrenamiento que predicen un alto $\mathrm{CC}$ en los hombres de nuestra población se pueda aplicar para mejorar el compromiso con los programas de actividad físico-deportiva en la población general.

Para estudios futuros, queda abierto un interesante campo de investigación para tratar de aplicar y evaluar la solidez y validez de estas conclusiones en otras poblaciones de practicantes de actividad físico-deportiva.

\section{Referencias}

Águila Soto, C. (2005). Ocio, jóvenes y posmodernidad. Almería: Universidad de Almería.

Ardila, R. (2003). Calidad de vida: una definición integradora. Revista Latinoamericana de Psicología, 35(2), 161-164.

Carmack, M. A. \& Martens, R. (1979). Measuring commitment to running: A survey of runner's attitudes and mental status. Journal of Sport \& Exercise Psychology, 1, 25-42.
Chapman, C. L. \& De Castro, J. M. (1990). Running addiction: Measurement and associated psychological characteristics. The Journal of Sports Medicine and Physical Fitness, 30, 283-290.

Dawson, K. A. \& Peco, J. (2004). Exercise motivation, commitment, addiction and gender. Medicine \& Science in Sports \& Exercise, 36, 64-65.

Fohelholm, M., Stallknecht, B. \& Van Baak, M. (2006). Exercise and obesity. European Journal of Sport Science, 6(1), 15-25.

García Ferrando, M. (2005). Encuesta sobre hábitos deportivos de los españoles 2005. Avance de resultados. Madrid: Ministerio de Educación, Cultura y Deporte/ Consejo Superior Deportes.

García Ureta, I. (2007). Addictive buying: Causes, processes, and symbolic meanings. Thematic analysis of a buying addict's diary. The Spanish Journal of Psychology, 10, 408-422.

Glasser, W. (1976). Positive addiction. New York: Harper $\&$ Row.

Gómez López, M., Ruiz-Juan, F., García Montes, M. E., Flores Allende, G. \& Barbero Montesinos, G. (2008). Razones que influyen en la inactividad físico-deportiva en la Educación Secundaria Post Obligatoria. Retos, 14, 80-85.

Kostrubala, T. (1977). The joy of running. New York: Simon \& Schuster.

Leedy, M. G. (2000). Commitment to distance running: Coping mechanism or addiction? Journal of Sport Behavior, 23, 255-270.

Masters, K. S., Ogles, B. M. \& Jolton, J. A. (1993). The development of an instrument to measure motivation for marathon running: The Motivations of Marathoners Scales (MOMS). Research Quarterly for Exercise and Sport, 64, 134-143.

Meeusen, R., Duclos, M., Gleeson, M., Rietjens, G., Steinacker, J. \& Urhausen, A. (2006). Prevention, diagnosis and treatment of the Overtraining Syndrome. European Journal of Sport Science, 6(1), 1-14.

Moreno Murcia, J. A., Martínez Galindo, C. \& Alonso Villodre, N. (2006). Actitudes hacia la práctica físico-deportiva según el sexo del practicante. Revista Internacional de Ciencias del Deporte, 2(3), 20-43.

Ogles, B. M. \& Masters, K. S. (2003). A typology of marathon runners based on cluster analysis of motivations. Journal of Sport Behavior, 26(1), 69-85. 
Pargman, D. (1980). The way of the runner: An examination of motives for running. En R. M. Suinn (Ed.), Psychology in sports: Methods and applications (pp. 90-98). Minneapolis: Burguess.

Real Academia Española. (2004). Diccionario de la lengua española (22....ed.) [Software de computadora en disco]. Madrid: Espasa-Calpe.

Ruiz-Juan, F. \& Zarauz, A. (2011a). Validación de la versión española de la Commitment to Running Scale (CR). Estudios de Psicología, 32(2), 195-207.

Ruiz-Juan, F. \& Zarauz, A. (2011b). Validación de la versión española de las Motivations of Marathoners Scales (MOMS). Revista Latinoamericana de Psicología, 43(1), 139-156.

Ruiz Tendero, G. (2006). El triatlón como modelo de sistema deportivo en el contexto nacional español e internacional: determinantes para su desarrollo y la consecución del éxito. Trabajo de grado, Universidad de Castilla-La Mancha, Toledo, España.
Thornton, E. W. \& Scott, S. E. (1995). Motivation in the committed runner: Correlations between self-report scales and behaviour. Health Promotion International, 10, 177-184.

Villamarín, F., Mauri, C. \& Sanz, A. (1998). Competencia percibida y motivación durante la iniciación en la práctica del tenis. Revista de Psicología del Deporte, 13, 41-56.

Valero Valenzuela, A., Gómez López, M., Gavala González, J., Ruiz-Juan, F. \& García Montes, M. E. (2007). ¿Por qué no se realiza actividad físicodeportiva en el tiempo libre? Motivos y correlatos sociodemográficos. Retos, 12, 13-17.

Vuori, I. (2004). Physical inactivity as a disease risk and health benefits of increased physical activity. Perspectives, 6, 1-73.

Zarauz, A. \& Ruiz-Juan, F. (2011). Propiedades psicométricas de la versión española de la Running Addiction Scale (RAS). The Spanish Journal of Psychology, 14(2), 967-976. 


\section{Apéndice 1}

\begin{tabular}{|c|c|c|c|c|c|}
\hline \multicolumn{6}{|l|}{ CR-11 (Ruiz-Juan \& Zarauz, 2011a) } \\
\hline \multicolumn{6}{|c|}{$\begin{array}{l}\text { Las siguientes frases pueden o no describir sus sentimientos sobre correr. Puntuar en una escala del } 1 \text { (totalmente en } \\
\text { desacuerdo) al } 5 \text { (totalmente de acuerdo) las siguientes afirmaciones que mejor describan la mayor parte del tiempo } \\
\text { sus sentimientos sobre correr. }\end{array}$} \\
\hline Estoy deseoso de correr & 1 & 2 & 3 & 4 & \\
\hline Correr es entretenido & 1 & 2 & 3 & 4 & 5 \\
\hline No disfruto corriendo (R) & 1 & 2 & 3 & 4 & 5 \\
\hline Correr es de vital importancia para mí & 1 & 2 & 3 & 4 & 5 \\
\hline Mi vida es mucho más rica porque corro & 1 & 2 & 3 & 4 & 5 \\
\hline Correr es agradable & 1 & 2 & 3 & 4 & 5 \\
\hline Me aterroriza la idea de correr $(\mathrm{R})$ & 1 & 2 & 3 & 4 & 5 \\
\hline Reorganizaría o cambiaría mi horario para satisfacer mi necesidad de correr & 1 & 2 & 3 & 4 & 5 \\
\hline Tengo que forzarme a mí mismo para ir a correr $(\mathrm{R})$ & 1 & 2 & 3 & 4 & 5 \\
\hline No ir un día a correr es un alivio enorme $(\mathrm{R})$ & 1 & 2 & 3 & 4 & 5 \\
\hline Correr es el punto culminante del día & 1 & 2 & 3 & 4 & 5 \\
\hline
\end{tabular}

(R) Estos ítems invierten su puntuación.

\section{Apéndice 2}

RAS-8 (Zarauz \& Ruiz-Juan, 2011).

Puntuar de 1 a 7 (el número 1 indica que no está nada de acuerdo con la afirmación que se realiza y el número 7 que estás totalmente de acuerdo) las siguientes afirmaciones al respecto de sus costumbres al correr.

\begin{tabular}{|c|c|c|c|c|c|c|c|}
\hline Si el clima es demasiado frío, caluroso o ventoso, no correré ese día (R) & 1 & 2 & 3 & 4 & 5 & 6 & 7 \\
\hline No cambiaría planes con amigos para poder ir a correr (R) & 1 & 2 & 3 & 4 & 5 & 6 & 7 \\
\hline $\begin{array}{l}\text { He dejado de correr al menos durante una semana por otra razón que no fue una } \\
\text { lesión (R) }\end{array}$ & 1 & 2 & 3 & 4 & 5 & 6 & 7 \\
\hline $\begin{array}{l}\text { Si hubiera otra manera de mantener mi forma física actual, no correría ninguna vez } \\
\text { más (R) }\end{array}$ & 1 & 2 & 3 & 4 & 5 & 6 & 7 \\
\hline Después de correr me siento mejor & 1 & 2 & 3 & 4 & 5 & 6 & 7 \\
\hline Continuaría corriendo mientras una lesión se me cura & 1 & 2 & 3 & 4 & 5 & 6 & 7 \\
\hline Algunos días, incluso si no me apetece correr, lo hago de todas maneras & 1 & 2 & 3 & 4 & 5 & 6 & \\
\hline Siento que necesito correr al menos una vez todos los días & 1 & 2 & 3 & 4 & 5 & 6 & 7 \\
\hline
\end{tabular}

(R) Estos ítems invierten su puntuación. 


\section{Apéndice 3}

\begin{tabular}{|c|c|c|c|c|c|c|c|}
\hline \multicolumn{8}{|c|}{ MOMS-34 (Ruiz-Juan \& Zarauz, 2011b) } \\
\hline \multicolumn{8}{|c|}{$\begin{array}{l}\text { Por favor, califique cada uno de los siguientes motivos para determinar la importancia de cada uno de ellos en la decisión de } \\
\text { por qué corre usted, considerando que una puntuación de } 1 \text { indicaría que para usted ese motivo no es una razón para correr, } \\
\text { hasta una puntuación de } 7 \text { que indicaría que ese motivo es una razón muy importante para correr. }\end{array}$} \\
\hline Para ayudar a controlar mi peso & 1 & 2 & 3 & 4 & 5 & 6 & 7 \\
\hline Para competir con otros & 1 & 2 & 3 & 4 & 5 & 6 & 7 \\
\hline Para ganar el respeto de los compañeros & 1 & 2 & 3 & 4 & 5 & 6 & 7 \\
\hline Para adelgazar & 1 & 2 & 3 & 4 & 5 & 6 & 7 \\
\hline Para mejorar mi velocidad de carrera (rodajes) & 1 & 2 & 3 & 4 & 5 & 6 & 7 \\
\hline Para ganar el respeto de la gente & 1 & 2 & 3 & 4 & 5 & 6 & 7 \\
\hline Para socializar con otros corredores & 1 & 2 & 3 & 4 & 5 & 6 & 7 \\
\hline Para mejorar mi salud & 1 & 2 & 3 & 4 & 5 & 6 & 7 \\
\hline Para competir conmigo mismo & 1 & 2 & 3 & 4 & 5 & 6 & 7 \\
\hline Para tener algo en común con otras personas & 1 & 2 & 3 & 4 & 5 & 6 & 7 \\
\hline Para prolongar mi vida & 1 & 2 & 3 & 4 & 5 & 6 & 7 \\
\hline Para conocer gente & 1 & 2 & 3 & 4 & 5 & 6 & 7 \\
\hline Para que mi familia o amigos estén orgullosos de mí & 1 & 2 & 3 & 4 & 5 & 6 & 7 \\
\hline Para tener más propósitos en mi vida & 1 & 2 & 3 & 4 & 5 & 6 & 7 \\
\hline Para parecer más delgado & 1 & 2 & 3 & 4 & 5 & 6 & 7 \\
\hline Para intentar correr más rápido & 1 & 2 & 3 & 4 & 5 & 6 & 7 \\
\hline Para participar con mi familia o amigos & 1 & 2 & 3 & 4 & 5 & 6 & 7 \\
\hline Para sentirme realizado por completo & 1 & 2 & 3 & 4 & 5 & 6 & 7 \\
\hline Para reducir la probabilidad de padecer un infarto & 1 & 2 & 3 & 4 & 5 & 6 & 7 \\
\hline Para hacer mi vida más completa & 1 & 2 & 3 & 4 & 5 & 6 & 7 \\
\hline Para compartir un espíritu de equipo con otros corredores & 1 & 2 & 3 & 4 & 5 & 6 & 7 \\
\hline Porque es una experiencia emocional positiva & 1 & 2 & 3 & 4 & 5 & 6 & 7 \\
\hline Para sentirme orgulloso de mí mismo & 1 & 2 & 3 & 4 & 5 & 6 & 7 \\
\hline Para quedar con amigos & 1 & 2 & 3 & 4 & 5 & 6 & 7 \\
\hline Para tener espíritu de superación & 1 & 2 & 3 & 4 & 5 & 6 & 7 \\
\hline Para mejorar mi marca actual & 1 & 2 & 3 & 4 & 5 & 6 & 7 \\
\hline Para pasar tiempo solo y pensar/organizar mis cosas & 1 & 2 & 3 & 4 & 5 & 6 & 7 \\
\hline Para concentrarme en mis pensamientos & 1 & 2 & 3 & 4 & 5 & 6 & 7 \\
\hline Para resolver problemas & 1 & 2 & 3 & 4 & 5 & 6 & 7 \\
\hline Para prevenir enfermedades & 1 & 2 & 3 & 4 & 5 & 6 & 7 \\
\hline Para que la gente se fije en mí & 1 & 2 & 3 & 4 & 5 & 6 & 7 \\
\hline Para ver si puedo batir una cierta marca & 1 & 2 & 3 & 4 & 5 & 6 & 7 \\
\hline Para conseguir reconocimiento/prestigio & 1 & 2 & 3 & 4 & 5 & 6 & 7 \\
\hline Para que los demás me elogien & 1 & 2 & 3 & 4 & 5 & 6 & 7 \\
\hline
\end{tabular}

Subescalas: Orientación a la salud: ítems 8, 11, 19 y 30. Peso: ítems 1, 4 y 15. Superación de metas personales-Competición: ítems 2, 5, 9, 16, 26 y 32. Reconocimiento: ítems 3, 6, 13, 31, 33 y 34. Afiliación: ítems 7, 10, 12, 17, 21 y 24. Meta psicológica: ítems 27, 28 y 29. Significado de la vida-Autoestima: ítems 14, 18, 20, 22, 23 y 25. 
